\title{
Mitral and Aortic Regurgitation in Patients Undergoing Kidney Transplantation: The Natural Course and Factors Associated With Progression
}

\author{
Minjeong Kim ${ }^{1}$, Darae Kim², Juhan Lee ${ }^{3}$, Dae-Young Kim ${ }^{1}$, Jiwon Seo ${ }^{1}$, Iksung Cho ${ }^{1}$, \\ Kyu Ha Huh ${ }^{3}$, Geu-Ru Hong ${ }^{1}$, Jong-Won $\mathrm{Ha}^{1}$ and Chi Young Shim ${ }^{1 *}$ \\ ${ }^{1}$ Division of Cardiology, Severance Cardiovascular Hospital, Yonsei University College of Medicine, Seoul, South Korea, \\ ${ }^{2}$ Division of Cardiology, Department of Medicine, Heart Vascular Stroke Institute, Samsung Medical Center, Sungkyunkwan \\ University School of Medicine, Seoul, South Korea, ${ }^{3}$ Departement of Surgery, Yonsei University College of Medicine, Seoul, \\ South Korea
}

OPEN ACCESS

Edited by:

Sanjeev Bhattacharyya Barts Heart Centre, United Kingdom

Reviewed by:

Sveeta Badiani,

Barts Health NHS Trust,

United Kingdom

Dimitrios Klettas,

Hippokration General Hospital, Greece

*Correspondence: Chi Young Shim

cysprs@yuhs.ac

Specialty section: This article was submitted to

Heart Valve Disease,

a section of the journa

Frontiers in Cardiovascular Medicine

Received: 05 November 2021 Accepted: 03 January 2022

Published: 27 January 2022

Citation:

Kim M, Kim D, Lee J, Kim D-Y, Seo J,

Cho I, Huh KH, Hong G-R, Ha J-W and Shim CY (2022) Mitral and Aortic Regurgitation in Patients Undergoing Kidney Transplantation: The Natural

Course and Factors Associated With Progression

Front. Cardiovasc. Med. 9:809707.

doi: 10.3389/fcvm.2022.809707
Background: Valve regurgitation can decrease with resolution of hemodynamic loads on the left ventricle (LV) after kidney transplantation (KT). We aimed to investigate the natural course of left-side valve regurgitation after KT and factors associated with progression.

Methods: Among patients who underwent KT in two tertiary centers, 430 (224 men, mean age $50 \pm 13$ years) were examined by echocardiography within 3 months before KT and between 6 and 36 months after KT. Mitral regurgitation (MR) and aortic regurgitation (AR) were graded according to the current guidelines. Regression was defined as a decrease in regurgitation by one or more steps, and progression was an increase in one or more steps after KT. Clinical and echocardiographic factors associated with progression of MR and AR were analyzed.

Results: Mild or greater MR was observed in 216 (50\%) patients before KT, and mild or greater AR was observed in 99 (23\%). During the follow-up period of $23.4 \pm 9.9$ months, most patients experienced regression or no change in regurgitation after KT, but 34 patients (7.9\%) showed MR progression and 37 (8.6\%) revealed AR progression. Patients who showed MR progression were more likely to receive a second KT, have mitral annular calcifications, and show a smaller decrease in LV end-systolic dimension. Patients who showed AR progression were more likely to have persistent hypertension after KT, aortic valve calcifications, and a smaller reduction of LV end-systolic dimension.

Conclusions: Risk factors for progression of MR after KT include a second KT, MAC and a smaller decrease in LV end-systolic dimension after KT. Risk factors for progression of AR include valve calcification, persistent hypertension and a smaller decrease in LV end-systolic dimension after KT. Further echocardiographic surveillance and risk factor management after KT are warranted in these patients.

Keywords: left-side valve disease, mitral regurgitation, aortic regurgitation, ESRD, kidney transplantation 


\section{INTRODUCTION}

Valve regurgitation is observed frequently in patients with chronic kidney disease or end stage renal disease (ESRD) (1, 2). Left-side valve regurgitation, including mitral regurgitation (MR) and aortic regurgitation (AR), is predicted to decrease when the hemodynamic load on the left ventricle (LV) decreases after kidney transplantation (KT). However, in some patients, left-side valve regurgitation does not decrease but persists or even progresses (3-5). In particular, degeneration and structural change of the valve start early in patients with ESRD because of the hemodynamic load and impaired calciumphosphate homeostasis and progress faster than in those with normal kidney function (6-8). MR and AR are affected by different hemodynamic factors and structural alterations. MR is influenced more highly by preload and LV remodeling, while AR is affected most by afterload and aorta remodeling. Patients who have undergone KT are thought to have reduced or increased MR or AR depending on the clinical situation in pre-KT and post-KT periods (9). However, data regarding the prevalence of left-side valve regurgitation in patients undergoing $\mathrm{KT}$ and the natural course of MR and AR after KT are scarce.

In the present study, we investigated the prevalence of MR and $\mathrm{AR}$ before $\mathrm{KT}$ and the regression or progression rate of MR and AR after KT. We also identified clinical and echocardiographic factors associated with progression of MR and AR.

\section{METHODS}

\section{Study Population}

This study included patients with ESRD who received KT between 2005 and 2018 at two tertiary medical centers (Severance Hospital, Seoul, Korea; Samsung Medical Center, Seoul, Korea). Patients with a history of valve surgery or intervention, congenital heart disease, or combined transplantation with other organs were excluded, as were patients without follow-up transthoracic echocardiography (TTE) after KT. After applying exclusion criteria, 430 patients were analyzed retrospectively.

All patients underwent TTE at least twice. Pre-KT echocardiography was performed within 3 months before $\mathrm{KT}$, and post-KT echocardiography was performed between 6 and 36 months after KT. If a patient underwent TTE more than twice after KT, we analyzed the most recent.

Clinical information was obtained from electronic medical records, and data were analyzed at baseline and follow-up TTE. Prior medical history was composed of hypertension, diabetes mellitus, dyslipidemia, atrial fibrillation, coronary artery disease, heart failure, duration of hemodialysis, and second KT. The diagnosis of heart failure was based on typical clinical symptoms and signs caused by a structural or functional cardiac abnormality, followed the current guideline, including both reduced EF and preserved EF (8). Systolic and diastolic blood pressures were measured at baseline and postKT follow-up visits. Clinical factors after KT including newonset hypertension, new-onset diabetes, renal dysfunction, graft failure, and cardiovascular medications were collected. Renal dysfunction was defined as a $>1.5$-fold increase in serum creatinine or an absolute increase in serum creatinine $\geq 0.3$ $\mathrm{mg} / \mathrm{dL}(9)$.

\section{Echocardiography}

Two-dimensional and Doppler echocardiography was performed using a commercially available ultrasound machine with a 2.5-3.5 $\mathrm{MHz}$ probe before and after KT. Standard measurements were performed following the current guideline recommendations (10). LV ejection fraction (EF) was measured using the biplane Simpson's method in apical four- and two-chamber views. Left atrial (LA) volume index was measured by the biplane method at the end of ventricular systole and indexed to body surface area. From the mitral inflow velocities, we obtained data on peak velocity of early (E) and late filling and deceleration time of $\mathrm{E}$ velocity. Early diastolic ( $\left.\mathrm{e}^{\prime}\right)$ velocities were measured at the septal mitral annulus (11).

The severity and mechanism of each valve regurgitation were assessed according to American Society of Echocardiography guidelines (12). The degrees of MR and AR, if present, were graded as no/trivial, mild, moderate, or severe using an integrated approach (12). The etiology of MR was categorized into primary or secondary MR. Primary MR was defined as degenerative MR directly affecting the mitral valve leaflets and/or chordae, and secondary MR was MR due to a pathological process of the LV or LA (12). Mitral annular calcification (MAC) was defined as the thick and echo-dense area of the mitral annulus, occasionally extending to mitral valve leaflets, as described in previous studies $(13,14)$. Aortic valve calcification was defined as a calcium deposit in the aortic root and valve regardless of restriction of leaflet mobility on parasternal short- and long-axis views $(15,16)$. The echocardiography reports were independently reviewed by 2 cardiologists blinded to the clinical data. All discrepancy of echocardiographic readings was resolved by consensus. In a consensus process, the 2 cardiologists reviewed the echocardiographic images and reached an agreement on the interpretation.

Regression of MR or AR was noted if severity decreased by one or more grades. Progression was defined as severity increase by one or more grades. Patients were categorized into three groups (regression, unchanged, progression) according to valve regurgitation.

\section{Statistical Analysis}

The baseline characteristics are expressed using frequencies and percentages for categorical variables using chi-square test. The continuous variables are summarized as mean \pm standard deviation and were compared using analysis of variance (ANOVA) among the three groups. For pairwise comparisons, post-hoc test with Tukey's HSD was conducted. To identify factors associated with progression of valve regurgitation, a linear regression model was used. Univariable factors with $P<0.10$ or the major relevant clinical factors were entered into multivariable analyses. The coefficient values were generated, and all twosided $p$-values $<0.05$ were considered statistically significant. The Hosmer-Lemeshow test for stepwise logistic regression was performed for incremental value of risk prediction. All statistical 


\section{Mitral Regurgitation}

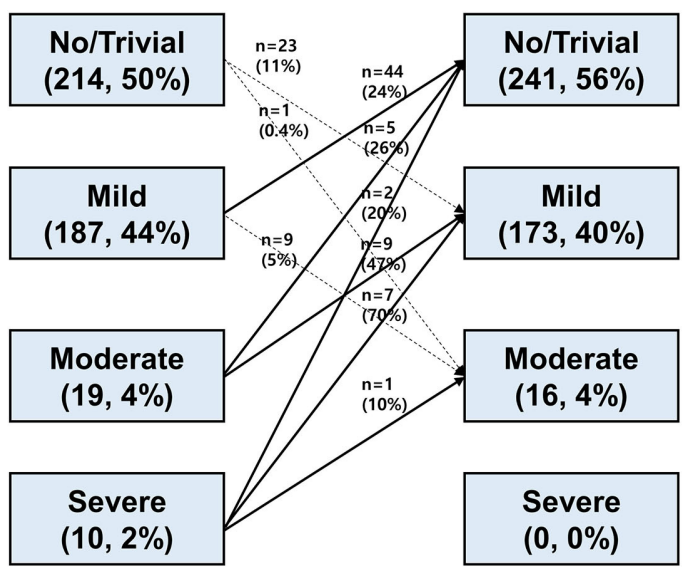

Before KT

After KT

\section{Aortic Regurgitation}

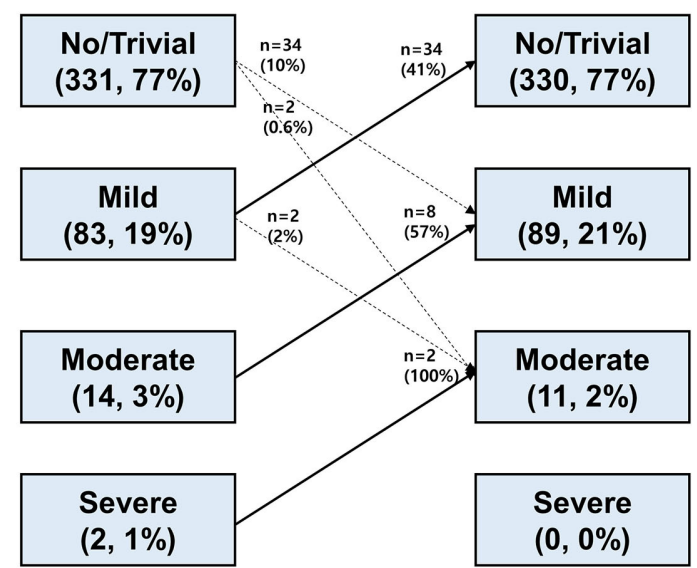

Before KT

FIGURE 1 | Natural course of left-sided valvular regurgitation after kidney transplantation.

analyses were performed using SPSS software (version 25.0; IBM Corp., Armonk, NY, USA).

\section{RESULTS}

\section{Severity and Changes of Left-Side Valve Regurgitation After KT}

The severity of MR and AR before KT and the changes after KT are shown in Figure 1. Before KT, 187 (44\%) patients had mild MR, 19 (4\%) had moderate MR, and 10 (2\%) showed severe MR. Factors related to MR severity before KT are presented in Supplementary Table 1. Female sex, history of heart failure, and larger LA volume index were associated with MR before KT. Before KT, 83 (19\%) patients had mild AR, 14 (3\%) had moderate $\mathrm{AR}$, and $2(1 \%)$ showed severe AR. Factors associated with AR severity before KT are described in Supplementary Table 2. Old age and history of heart failure were associated with AR grade before KT. Mild or greater MR was observed in 216 (50\%) patients and mild or greater AR in 99 (23\%) before KT. During the follow-up period of $23.4 \pm 9.9$ months, most patients experienced regression or no change in regurgitation after $\mathrm{KT}$, but $34(7.9 \%)$ showed MR progression and 37 (8.6\%) revealed AR progression.

\section{Factors Associated With MR Progression}

Table 1 shows the baseline clinical and renal characteristics in the three groups according to change in MR after KT. A history of atrial fibrillation, history of heart failure and secondary MR tended to be more prevalent in the MR regression group compared with the other groups. Second KT and post-KT renal dysfunction were significantly different in the three groups, and these events tended to be more prevalent in the progression group. Most of the echocardiographic findings differed between the three groups. In patients with MR progression, LV chamber size was small, and E/e' was lower than that in the other groups before KT, but these factors were significantly higher after KT. In the group showing MR progression, LV and LA size increased after KT, LVEF decreased, and E/e' increased (Table 2).

Univariate linear regression analysis revealed that second $\mathrm{KT}$, MAC before KT, renal dysfunction after KT, and a smaller decrease in LV end systolic dimension (LVESD) were associated with MR progression. After multivariable adjustment, second KT, baseline MAC, and smaller decrease in LVESD after KT were independent predictors for MR progression after KT (Table 3). Factors related to progression of MR showed statistically meaningful predictive values in a stepwise manner (Figure 2A).

These findings indicate that MR tended to show regression after KT for controlling volume overload. In contrast, MR progression can occur after KT if the volume overload is relatively low before $\mathrm{KT}$ or if valve degeneration represented by MAC is present before KT. Furthermore, progression of MR was related to subsequent elevation of LV filling pressure after KT.

\section{Factors Associated With AR Progression}

Table 4 shows the baseline clinical and renal characteristics of the three groups categorized by AR change after KT. Patients who showed AR progression experienced persistent hypertension, defined as systolic blood pressure $\geq 140 \mathrm{mmHg}$ and diastolic blood pressure $\geq 90 \mathrm{mmHg}$ after KT among those with hypertension. In echocardiographic findings, patients who showed AR progression showed larger LV and LA size after KT compared with the other groups. Similar to the results from the analysis of MR, smaller reductions in LV and LA sizes after KT, no improvement in LVEF, and an increase in E/e' were observed in the AR progression group (Table 5). 
TABLE 1 | Comparison of clinical and renal characteristics in the three groups according to change in mitral regurgitation.

\begin{tabular}{|c|c|c|c|c|}
\hline & $\begin{array}{c}\text { MR } \\
\text { regression } \\
(n=68)\end{array}$ & $\begin{array}{c}\text { MR } \\
\text { unchanged } \\
(n=328)\end{array}$ & $\begin{array}{c}\text { MR } \\
\text { progression } \\
(n=34)\end{array}$ & $p$-value \\
\hline \multicolumn{5}{|l|}{ Clinical characteristics } \\
\hline Age, years & $51.0 \pm 11.6$ & $49.5 \pm 13.0$ & $53.9 \pm 10.7$ & 0.128 \\
\hline Male sex, $n(\%)$ & $28(40.6)$ & $180(55.0)$ & $16(47.1)$ & 0.076 \\
\hline Hypertension, $n$ (\%) & $58(84.1)$ & $299(91.7)$ & $29(85.3)$ & 1.000 \\
\hline Diabetes mellitus, $n(\%)$ & $24(34.8)$ & $114(35.0)$ & $16(47.1)$ & 0.368 \\
\hline Dyslipidemia, $n$ (\%) & $16(23.2)$ & $62(19.0)$ & $3(8.8)$ & 0.214 \\
\hline Atrial fibrillation, $n(\%)$ & $9(13.0)$ & $13(4.0)^{\star}$ & $0(0.0)^{\#}$ & 0.003 \\
\hline CAD, $n(\%)$ & $9(13.0)$ & $37(11.3)$ & $7(20.6)$ & 0.292 \\
\hline Heart failure, $n(\%)$ & $13(18.8)$ & $24(7.3)^{\star}$ & $2(5.9)^{\#}$ & 0.008 \\
\hline \multicolumn{5}{|l|}{ Renal characteristics } \\
\hline Dialysis, $n$ (\%) & $68(100.0)$ & $328(100.0)$ & $34(100.0)$ & 1.000 \\
\hline Hemodialysis, $n$ (\%) & $62(91.2)$ & $282(86.0)$ & $31(91.2)$ & 0.664 \\
\hline $\begin{array}{l}\text { Peritoneal dialysis, } n \\
(\%)\end{array}$ & $6(8.8)$ & $46(14.0)$ & $3(8.8)$ & 0.306 \\
\hline HD duration, months & $56.7 \pm 58.2$ & $65.2 \pm 66.9$ & $56.4 \pm 62.1$ & 0.577 \\
\hline Second KT, $n(\%)$ & $2(2.9)$ & $23(7.0)$ & $7(20.6)^{\star \#}$ & 0.005 \\
\hline \multicolumn{5}{|c|}{ Post-KT comorbidities, $n$ (\%) } \\
\hline New-onset HTN & $4(5.8)$ & $13(4.0)$ & $2(5.9)$ & 0.732 \\
\hline Persistent HTN & $13(18.8)$ & $103(31.5)^{\star}$ & $17(50.0)^{*} \#$ & 0.005 \\
\hline New-onset DM & $5(7.2)$ & $52(16.0)$ & $3(8.8)$ & 0.111 \\
\hline $\begin{array}{l}\text { Renal dysfunction, } n \\
\text { (\%) }\end{array}$ & $9(13.0)$ & $62(19.0)$ & $12(35.3)^{*} \#$ & 0.025 \\
\hline Graft failure, $n(\%)$ & $11(15.9)$ & $33(10.1)$ & $6(17.6)$ & 0.202 \\
\hline \multicolumn{5}{|l|}{ Post-KT medications } \\
\hline RAAS blocker, $n(\%)$ & $12(18.8)$ & $74(26.9)$ & $5(15.6)$ & 0.186 \\
\hline Beta blocker, $n$ (\%) & $23(34.8)$ & $145(52.3)^{\star}$ & $14(43.8)$ & 0.032 \\
\hline $\mathrm{CCB}, n(\%)$ & $25(36.0)$ & $108(32.9)$ & $17(50.0)$ & 0.470 \\
\hline Diuretics, $n(\%)$ & $6(9.4)$ & $27(9.9)$ & $4(12.1)$ & 0.906 \\
\hline Statin, $n(\%)$ & $17(26.2)$ & $85(31.4)$ & $12(36.4)$ & 0.556 \\
\hline
\end{tabular}

${ }^{*} P<0.05$ compared with the MR regression group.

${ }^{\#} P<0.05$ compared with the MR unchanged group.

$M R$, mitral regurgitation; $C A D$, coronary artery disease; $H D$, hemodialysis; $K T$, kidney transplantation; HTN, hypertension; DM, diabetes mellitus; RAAS, renin-angiotensinaldosterone system; CCB, calcium channel blocker.

Univariate linear regression analysis revealed that presence of aortic valve calcification before KT, persistent HTN after KT, and a smaller decrease in LVESD after KT were associated with AR progression. All the above factors were independent predictors for AR progression after KT in multivariate analysis (Table 6). Figure 2B shows additive predictive values for AR progression after KT in a stepwise manner. These findings suggest that preexisting aortic valve degeneration accompanying unresolved afterload as well as less volume control after KT might affect AR progression.

\section{DISCUSSION}

The principal findings in the present multicenter study are as follows. (1) Left-side valve regurgitation is common in patients
TABLE 2 | Comparison of echocardiographic characteristics in the three groups according to change in mitral regurgitation.

$\begin{array}{cccc}\begin{array}{c}\text { MR } \\ \text { regression } \\ (n=68)\end{array} & \begin{array}{c}\text { MR } \\ \text { unchanged }\end{array} & \begin{array}{c}\text { MR } \\ \text { progression }\end{array} & p \text {-value } \\ & (n=328) & (n=34) & \end{array}$

Pre-KT echocardiogram

$\begin{array}{lcccc}\text { LVEDD, mm } & 56.6 \pm 6.9 & 52.4 \pm 5.9 & 51.3 \pm 5.7^{*} & <0.001 \\ \text { LVESD, mm } & 38.5 \pm 9.3 & 34.1 \pm 6.0 & 62.5 \pm 6.1^{\star} & <0.001 \\ \text { LVEF, \% } & 56.7 \pm 12.8 & 62.3 \pm 9.3 & 61.7 \pm 10.6 & <0.001 \\ \text { LV mass index, g/m² } & 148.4 \pm 51.1 & 124.9 \pm 37.0 & 118.6 \pm 36.4 & <0.001 \\ \text { LA volume index, } & 51.6 \pm 24.5 & 38.1 \pm 15.1 & 39.5 \pm 14.8 & <0.001 \\ \text { ml/m }{ }^{2} & & & & \\ \text { E/e' } & 15.4 \pm 6.9 & 12.4 \pm 5.6^{\star} & 12.2 \pm 4.1^{*} & 0.001 \\ \text { PASP, mmHg } & 40.0 \pm 11.8 & 30.3 \pm 9.3^{\star} & 35.4 \pm 10.5 & 0.015 \\ \text { Degree of MR, } n(\%) & & & & <0.001 \\ \text { No/trivial } & 0(0) & 190(57.8)^{\star} & 24(70.6)^{* \#} & \\ \text { Mild } & 46(66.7) & 131(40.1)^{\star} & 10(29.4)^{\star} & \\ \text { Moderate } & 12(17.4) & 7(2.1)^{\star} & 0(0.0)^{\star} & \\ \text { Severe } & 10(14.5) & 0(0.0)^{\star} & 0(0.0)^{\star} & \\ \text { Secondary MR, } n(\%) & 68(100.0) & 111(76.5)^{\star} & 28(82.3)^{\star} & <0.001 \\ \text { Presence of MAC, } n & 6(8.7) & 19(5.8) & 6(17.6)^{\#} & 0.035\end{array}$

(\%)

Post-KT echocardiogram

$\begin{array}{lcccc}\text { LVEDD, mm } & 48.7 \pm 5.6 & 48.9 \pm 5.8 & 52.0 \pm 5.8 & 0.011 \\ \text { LVESD, mm } & 30.3 \pm 5.6 & 30.4 \pm 5.1 & 33.5 \pm 5.7 & 0.006 \\ \text { LVEF, \% } & 65.4 \pm 7.6 & 65.8 \pm 7.8 & 58.4 \pm 9.6 & <0.001 \\ \text { LV mass index, g/m² } & 108.3 \pm 34.4 & 111.7 \pm 33.5 & 125.6 \pm 33.9 & 0.045 \\ \text { LA volume index, } & 36.8 \pm 14.4 & 35.5 \pm 16.3 & 45.0 \pm 17.8 & 0.006 \\ \mathrm{ml} / \mathrm{m}^{2} & & & & \\ \text { E/e' } & 11.6 \pm 5.1 & 11.5 \pm 4.8 & 16.6 \pm 7.2^{\star \#} & <0.001 \\ \text { PASP, mmHg } & 30.3 \pm 11.6 & 29.5 \pm 7.9 & 42.7 \pm 13.4^{\star \#} & 0.003 \\ \text { Degree of MR, } n(\%) & & & & <0.001 \\ \text { No/trivial } & 51(73.9) & 190(58.1)^{\star} & 0(0.0)^{\star \#} & \\ \text { Mild } & 17(24.6) & 132(40.4)^{\star} & 24(70.6)^{\star \#} & \\ \text { Moderate } & 1(1.4) & 5(1.5) & 10(29.4)^{\star \#} & \\ \text { Severe } & 0(0.0) & 0(0.0) & 0(0.0) & \end{array}$

\section{Changes after KT}

\begin{tabular}{lcccc}
$\Delta$ LVEDD, $\mathrm{mm}$ & $-1.8 \pm 9.6$ & $1.1 \pm 9.4$ & $0.9 \pm 5.8$ & 0.082 \\
$\Delta$ LVESD, $\mathrm{mm}$ & $-8.1 \pm 8.8$ & $-3.7 \pm 5.4^{\star}$ & $0.9 \pm 6.5^{*}$ & $<0.001$ \\
$\Delta$ LVEF, \% & $8.3 \pm 13.6$ & $3.5 \pm 8.4$ & $-3.3 \pm 10.6^{*}$ & $<0.001$ \\
$\Delta$ E/e' & $-4.4 \pm 6.5$ & $-1.1 \pm 5.4^{\star}$ & $4.2 \pm 5.3^{\star \#}$ & $<0001$ \\
$\Delta$ LV mass index, $\mathrm{g} / \mathrm{m}^{2}$ & $-42.8 \pm 53.4$ & $-12.5 \pm 46.6$ & $7.4 \pm 42.7^{\star \#}$ & $<0.001$ \\
$\begin{array}{l}\Delta \text { LA volume index, } \\
\mathrm{ml} / \mathrm{m}^{2}\end{array}$ & $-15.7 \pm 24.7$ & $-4.7 \pm 13.8$ & $1.0 \pm 17.7^{*}$ & $<0.001$ \\
\hline
\end{tabular}

${ }^{*} P<0.05$ compared with the MR regression group.

${ }^{\#} P<0.05$ compared with the MR unchanged group.

$M R$, mitral regurgitation; $L V E D D$, left ventricular end-diastolic diameter; LVESD, lefMRt ventricular end-systolic diameter; $L V E F$, left ventricular ejection fraction; $L V$, left ventricle; $L A$, left atrium; E/e', ratio of early diastolic mitral velocity to early diastolic mitral annular velocity; PASP, pulmonary arterial systolic pressure; MAC, mitral annular calcification.

with ESRD undergoing $\mathrm{KT}$, and MR is more common than AR. (2) After KT, MR and AR regress or do not change in most patients but progress in some patients. (3) Patients who receive second $\mathrm{KT}$ and who have MAC and a smaller decrease in LVESD 
TABLE 3 | Factors associated with progression of mitral regurgitation after KT.

\begin{tabular}{|c|c|c|c|c|c|c|}
\hline & \multicolumn{3}{|c|}{ Univariate analysis } & \multicolumn{3}{|c|}{ Multivariate analysis } \\
\hline & $B$ & $t$ & $p$-value & $B$ & $t$ & $p$-value \\
\hline Age & 0.002 & 1.82 & 0.070 & 0.001 & 1.29 & 0.198 \\
\hline Female sex & 0.016 & 0.61 & 0.541 & & & \\
\hline Hypertension & -0.041 & -0.95 & 0.345 & & & \\
\hline Diabetes mellitus & 0.038 & 1.41 & 0.158 & & & \\
\hline Dyslipidemia & -0.052 & -1.56 & 0.119 & & & \\
\hline Atrial fibrillation & -0.084 & -1.41 & 0.158 & & & \\
\hline CAD & 0.060 & 1.52 & 0.129 & & & \\
\hline Heart failure & -0.031 & -0.67 & 0.501 & & & \\
\hline HD duration & -0.032 & -0.59 & 0.558 & & & \\
\hline Second KT & 0.151 & 3.07 & 0.002 & 0.114 & 2.31 & 0.022 \\
\hline New-onset HTN & 0.027 & 0.43 & 0.669 & & & \\
\hline Persistent HTN & 0.036 & 1.21 & 0.228 & & & \\
\hline New-onset DM & -0.034 & -0.90 & 0.367 & & & \\
\hline Renal dysfunction & 0.081 & 2.47 & 0.014 & 0.062 & 1.19 & 0.057 \\
\hline Graft failure & 0.046 & 1.14 & 0.255 & & & \\
\hline Secondary MR & -0.068 & -1.07 & 0.286 & & & \\
\hline Presence of MAC & 0.123 & 2.46 & 0.014 & 0.110 & 2.20 & 0.028 \\
\hline$\Delta$ LVEDD & 0.008 & 0.16 & 0.876 & & & \\
\hline$\Delta$ LVESD & 0.009 & 4.57 & $<0.001$ & 0.008 & 4.15 & $<0.001$ \\
\hline$\Delta$ LVEF & -0.006 & -4.21 & $<0.001$ & & & \\
\hline$\Delta \mathrm{LV}$ mass index & 0.001 & 2.79 & 0.006 & & & \\
\hline$\Delta$ LA volume index & 0.002 & 2.43 & 0.016 & & & \\
\hline
\end{tabular}

CAD, coronary artery disease; $H D$, hemodialysis; KT, kidney transplantation; HTN, hypertension; DM, diabetes mellitus; MR, mitral regurgitation; MAC, mitral annular calcification; $L V E D D$, left ventricular end-diastolic diameter; $L V E S D$, left ventricular endsystolic diameter; $L V E F$, left ventricular ejection fraction; $L V$, left ventricle; $L A$, left atrium. after KT showed MR progression after KT. (4) Patients who had aortic valve calcification, persistent HTN after KT, and a smaller decrease in LVESD after KT showed AR progression. The present study suggests that pre-existing valve degeneration before $\mathrm{KT}$, smaller volume changes, and specific conditions before or after KT affect the progression of regurgitation on each valve. As expected, MR was affected more highly by volume factor, and AR was affected most by afterload, such as persistent HTN after KT. Therefore, it is necessary to perform echocardiographic surveillance and risk factor control after KT according to individual characteristics before and after KT and presence of valve calcification.

\section{Left-Side Valve Disease in Patients With ESRD}

Patients with chronic kidney disease or ESRD show a high prevalence of left-side valve disease due to not only degeneration of the valve itself, but also chamber dilatation or dysfunction related with increased hemodynamic load and loss of LV contractility $(8,17)$. Previous studies demonstrated higher prevalence of premature aortic valve calcification and consequent aortic stenosis, as well as mitral annular calcification and functional mitral stenosis (18-21). In particular, increased calcium $\mathrm{x}$ phosphate product and long-term hemodialysis are associated with valve calcification $(8,17)$. Also, increased cardiac output caused by anemia and arteriovenous fistula and hypertension increase mechanical stress in the valve leaflets and modulate premature valve calcification $(8,17)$. In stenotic valve disease, primary degeneration is the main mechanism, whereas regurgitation is caused mainly by secondary causes. Therefore, because of preload and afterload reduction after KT

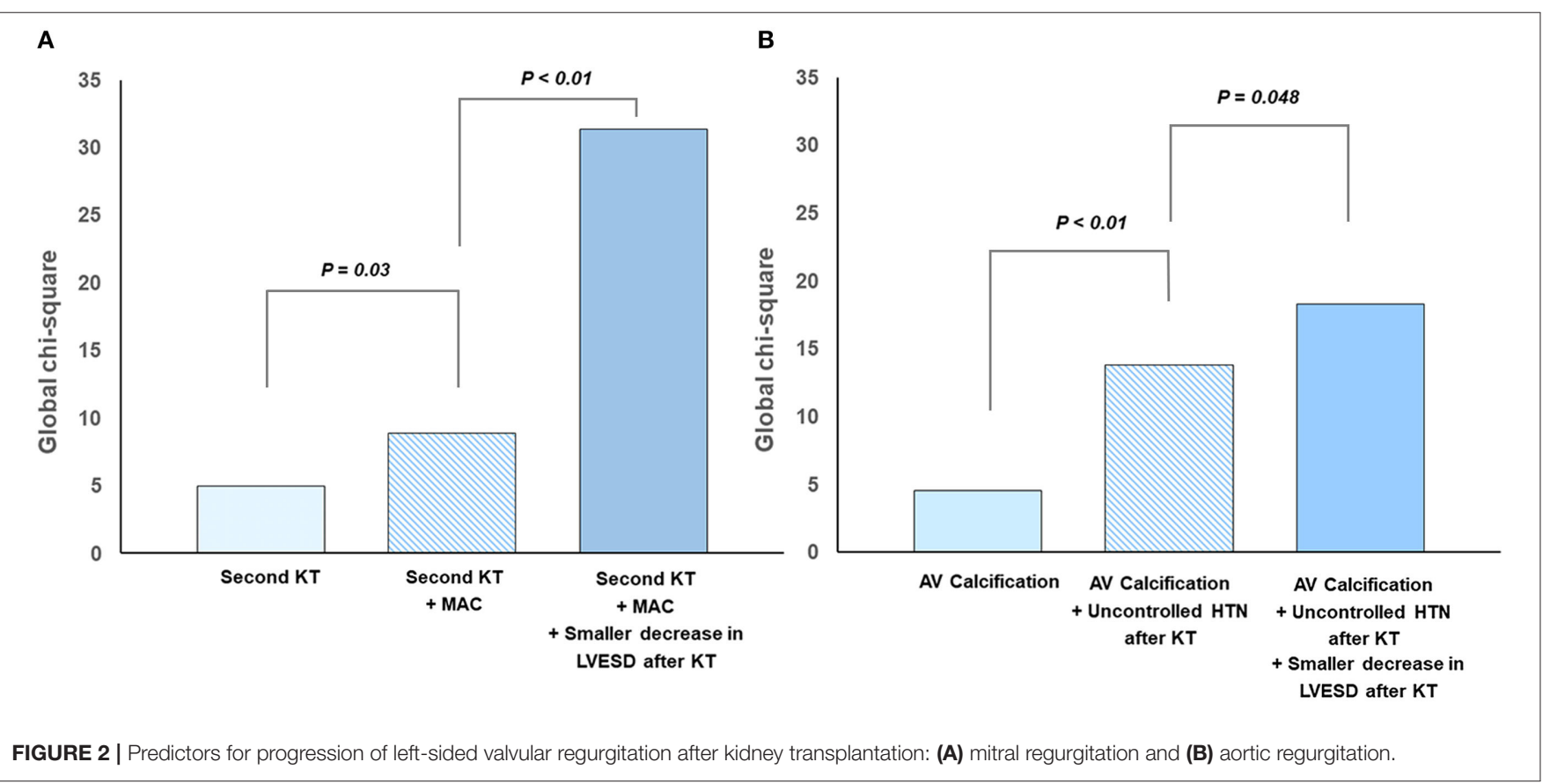


and additional improvement of renin-angiotensin-aldosterone system (RAAS) activation, regression of valve regurgitation is expected after KT. If the patient does not show any specific clinical features after KT, regular echocardiographic follow-up is not performed; valve regurgitation sometimes progresses, and timely treatment can be delayed.

\section{Natural Course of MR and AR in Patients Undergoing KT}

Several previous studies have focused on valve disease in patients undergoing KT. A serial echocardiographic follow-up study in 95 patients undergoing KT reported no interval change in average MR fraction and volume after KT (22). However, this study was limited in that it did not provide information on patient characteristics or changes in chamber size and function. Another study conducted at a single center with 180 patients undergoing KT demonstrated that grade 2 MR decreased from $11 \%$ on pre-KT echocardiogram to $2 \%$ at 12 months after KT (23). Interestingly, valve calcification was detected preoperatively in $21.5 \%$ of the study population but was detected in $25.8 \%$ of the population at 6 months after KT and in $35.5 \%$ of the population 12 months after KT (23). Incidence of calcified valve was more common in patients with diabetes than in those without it (23). These results suggest that, even if hemodynamic load is resolved by KT, valve degeneration and calcification can have initiated and can proceed. It also suggests that risk factor modulation and echocardiographic follow-up are needed even after KT in patients who have calcification on the valve or surrounding annular structure. In this context, the present study showed on pre-KT echocardiogram that MR and AR can progress even after $\mathrm{KT}$ in patients with MAC or aortic valve calcification through a multicenter analysis. In addition, we found some differential risk factors between MR progression and AR progression after KT.

\section{Risk Factors for Progression of MR and AR After KT}

Smaller LVESD decrease after KT was related to progression of not only MR, but also AR in this study. Therefore, a smaller volume decrease after KT was a pre-condition of progression of left-side valve regurgitation. These results indicate the need for follow-up TTE for surveillance of chamber size and course of functional regurgitation after KT, especially for patients who have predisposing factors. Furthermore, prolonged optimal medical treatment might be necessary after KT, especially for patients with risk factors of progression. In our study, there might have been insufficient usage of RAAS inhibitor in patients with progression of AR compared with that in the unchanged AR group. Since RASS inhibitor can promote reverse LV remodeling after KT through both afterload reduction and inhibition of myocardial fibrosis, active use of RASS inhibitor after KT seems to be helpful for hemodynamic control.

MAC or aortic valve calcification shares biological links with atherosclerosis and is very common in patients with ESRD (18). Patients with valve or peri-valvular calcification in ESRD were more likely to have dyslipidemia and a broken mineral-bone axis, and this condition might not recover fully after KT (16).
TABLE 4 | Comparison of clinical and renal characteristics in the three groups according to change in aortic regurgitation.

\begin{tabular}{|c|c|c|c|c|}
\hline & $\begin{array}{c}\text { AR } \\
\text { regression } \\
(n=38)\end{array}$ & $\begin{array}{c}\text { AR } \\
\text { unchanged } \\
(n=355)\end{array}$ & $\begin{array}{c}\text { AR } \\
\text { progression } \\
(n=37)\end{array}$ & $p$-value \\
\hline \multicolumn{5}{|l|}{ Clinical characteristics } \\
\hline Age, years & $53.2 \pm 12.9$ & $49.4 \pm 12.8$ & $53.7 \pm 9.8$ & 0.044 \\
\hline Male sex, $n(\%)$ & $11(28.9)$ & $198(55.8)^{\star}$ & $15(40.5)$ & 0.002 \\
\hline Hypertension, $n$ (\%) & $32(84.2)$ & $320(90.4)$ & $34(91.9)$ & 0.445 \\
\hline Diabetes mellitus, $n$ (\%) & $14(36.8)$ & $124(35.0)$ & $16(43.2)$ & 0.607 \\
\hline Dyslipidemia, $n$ (\%) & $6(15.8)$ & $71(20.1)$ & $4(10.8)$ & 0.345 \\
\hline Atrial fibrillation, $n(\%)$ & $5(13.2)$ & $16(4.5)$ & $1(2.7)$ & 0.056 \\
\hline CAD, $n(\%)$ & $6(15.8)$ & $43(12.1)$ & $4(10.8)$ & 0.775 \\
\hline Heart failure, $n(\%)$ & $8(21.1)$ & $28(7.9)^{\star}$ & $3(8.1)$ & 0.027 \\
\hline \multicolumn{5}{|l|}{ Renal characteristics } \\
\hline Dialysis, $n$ (\%) & 38 (100.0) & 355 (100.0) & 37 (100.0) & 1.000 \\
\hline Hemodialysis, $n$ (\%) & $35(92.1)$ & 305 (85.9) & 35 (94.6) & 0.473 \\
\hline Peritoneal dialysis, $n$ (\%) & $3(7.9)$ & $50(14.1)$ & $2(5.4)$ & 0.519 \\
\hline HD duration, months & $62.9 \pm 64.6$ & $64.3 \pm 66.6$ & $55.0 \pm 54.9$ & 0.733 \\
\hline Second KT, n (\%) & $4(10.5)$ & $24(6.8)$ & $4(10.8)$ & 0.503 \\
\hline \multicolumn{5}{|c|}{ Post-KT comorbidities, $n(\%)$} \\
\hline New-onset HTN & $3(7.9)$ & $15(4.2)$ & $1(2.7)$ & 0.504 \\
\hline Persistent HTN & $9(23.7)$ & $103(29.0)$ & $21(56.8)^{\star \#}$ & 0.001 \\
\hline New-onset DM & $3(7.9)$ & $54(15.3)$ & $3(8.1)$ & 0.258 \\
\hline Renal dysfunction, $n(\%)$ & $4(10.5)$ & $69(19.4)$ & $10(27.0)$ & 0.192 \\
\hline Graft failure, $n(\%)$ & $10(26.3)$ & $33(9.3)^{\star}$ & $7(18.9)$ & 0.003 \\
\hline \multicolumn{5}{|c|}{ Post-KT medications, $\boldsymbol{n}$ (\%) } \\
\hline RAAS blocker & $2(5.2)$ & $91(30.3)^{\star}$ & $3(8.1)^{\#}$ & $<0.001$ \\
\hline Beta blocker & $16(43.2)$ & $149(49.5)$ & $17(45.9)$ & 0.731 \\
\hline CCB & $16(34.0)$ & $267(33.7)$ & $20(37.0)$ & 0.630 \\
\hline Diuretics & $4(10.8)$ & $32(10.8)$ & $1(2.7)$ & 0.296 \\
\hline Statin & $0(0)$ & $110(36.9)^{*}$ & $4(11.1)^{\star \#}$ & $<0.001$ \\
\hline
\end{tabular}

${ }^{\star} P<0.05$ compared with the AR regression group.

${ }^{\#} P<0.05$ compared with the AR unchanged group.

$A R$, aortic regurgitation; $C A D$, coronary artery disease; $H D$, hemodialysis; $K T$, kidney transplantation; HTN, hypertension; DM, diabetes mellitus; RAAS, renin-angiotensinaldosterone system; CCB, calcium channel blocker.

These conditions were thought to be due to valve dysfunction accompanied by valve calcification that could progress after KT. This might be related to greater use of statins in patients who showed progression of AR after KT.

After KT, hypertension remains widespread, with 56-93\% of recipients consistently having a systolic blood pressure $>140$ mmHg. Multiple factors can lead to hypertension, including donor and recipient characteristics, immunosuppressive medications, and allograft function (17). Long-term exposure to high blood pressure is a strong and potentially modifiable risk factor for aortic stenosis and regurgitation (19). Our study also revealed persistent HTN after KT as a predictor of AR progression. This suggests that unresolved afterload and preload affect AR progression; therefore, strict anti-hypertensive treatment is important for preventing progression of $\mathrm{AR}$ after KT. 
TABLE 5 | Comparison of echocardiographic characteristics in the three groups according to change in aortic regurgitation.

\begin{tabular}{|c|c|c|c|c|}
\hline & $\begin{array}{c}\text { AR } \\
\text { regression } \\
(n=38)\end{array}$ & $\begin{array}{c}\text { AR } \\
\text { unchanged } \\
(n=355)\end{array}$ & $\begin{array}{c}\text { AR } \\
\text { progression } \\
(n=37)\end{array}$ & $p$-value \\
\hline \multicolumn{5}{|l|}{ Pre-KT echocardiogram } \\
\hline LVEDD, mm & $55.6 \pm 8.0$ & $52.6 \pm 6.1^{*}$ & $53.7 \pm 4.5$ & 0.018 \\
\hline LVESD, mm & $36.4 \pm 10.2$ & $34.6 \pm 6.5$ & $32.9 \pm 5.4$ & 0.095 \\
\hline LVEF, \% & $58.7 \pm 13.5$ & $61.5 \pm 9.9$ & $63.4 \pm 8.8$ & 0.141 \\
\hline LV mass index, $\mathrm{g} / \mathrm{m}^{2}$ & $139.7 \pm 55.8$ & $127.0 \pm 39.0$ & $128.6 \pm 34.4$ & 0.195 \\
\hline $\begin{array}{l}\text { LA volume index, } \\
\mathrm{ml} / \mathrm{m}^{2}\end{array}$ & $54.1 \pm 29.8$ & $38.5 \pm 14.8^{\star}$ & $45.3 \pm 19.2$ & $<0.001$ \\
\hline$E / e^{\prime}$ & $14.9 \pm 6.9$ & $12.7 \pm 5.7$ & $12.6 \pm 5.1$ & 0.113 \\
\hline PASP, mmHg & $36.6 \pm 11.2$ & $30.0 \pm 10.3$ & $29.8 \pm 9.1$ & 0.006 \\
\hline Degree of $A R, n(\%)$ & & & & $<0.001$ \\
\hline No/trivial & $0(0)$ & $296(83.4)^{\star}$ & $35(94.6)^{\star}$ & \\
\hline Mild & $34(89.5)$ & $47(13.2)^{\star}$ & $2(5.4)^{\star \#}$ & \\
\hline Moderate & $4(10.5)$ & $10(2.8)^{\star}$ & $0(0)^{*}$ & \\
\hline Severe & $0(0)$ & $2(0.6)$ & $0(0)$ & \\
\hline AV calcification, $n(\%)$ & $4(10.5)$ & $33(9.3)$ & $8(21.6)^{\#}$ & 0.018 \\
\hline \multicolumn{5}{|c|}{ Post-KT echocardiogram } \\
\hline LVEDD, mm & $48.7 \pm 6.7$ & $48.8 \pm 5.8$ & $51.9 \pm 4.7^{\star \#}$ & 0.008 \\
\hline LVESD. Mm & $28.7 \pm 5.5$ & $30.7 \pm 5.3$ & $31.8 \pm 5.1^{*}$ & 0.033 \\
\hline LVEF, \% & $66.5 \pm 7.4$ & $65.2 \pm 8.2$ & $63.1 \pm 7.9$ & 0.185 \\
\hline LV mass index, $\mathrm{g} / \mathrm{m}^{2}$ & $112.9 \pm 34.9$ & $111.8 \pm 34.0$ & $116.7 \pm 31.7$ & 0.706 \\
\hline $\begin{array}{l}\text { LA volume index, } \\
\mathrm{ml} / \mathrm{m}^{2}\end{array}$ & $43.2 \pm 18.6$ & $35.0 \pm 15.4^{\star}$ & $44.0 \pm 19.5^{\#}$ & $<0.001$ \\
\hline E/e' & $12.0 \pm 6.5$ & $11.6 \pm 4.7$ & $14.7 \pm 7.1$ & 0.005 \\
\hline PASP, mmHg & $33.1 \pm 12.5$ & $28.7 \pm 9.9$ & $31.0 \pm 9.2$ & 0.083 \\
\hline Degree of AR, $n(\%)$ & & & & $<0.001$ \\
\hline No/trivial & $34(89.5)$ & $296(83.4)$ & $0(0)^{\star \#}$ & \\
\hline Mild & $4(10.5)$ & $51(14.4)$ & $34(91.9)^{\star \#}$ & \\
\hline Moderate & $0(0)$ & $8(2.2)$ & $3(8.1)^{\star \#}$ & \\
\hline Severe & $0(0)$ & $0(0)$ & $0(0)$ & \\
\hline \multicolumn{5}{|l|}{ Changes after KT } \\
\hline$\Delta$ LVEDD, mm & $-4.5 \pm 9.1$ & $1.4 \pm 9.7^{\star}$ & $-1.1 \pm 6.4$ & 0.001 \\
\hline$\Delta$ LVESD, mm & $-7.5 \pm 9.8$ & $-4.0 \pm 6.0^{*}$ & $-1.2 \pm 5.7^{\star \#}$ & $<0.001$ \\
\hline$\Delta$ LVEF, \%) & $7.3 \pm 13.6$ & $3.7 \pm 9.4$ & $-0.2 \pm 9.1^{\star \#}$ & 0.006 \\
\hline$\Delta E / e^{\prime}$ & $-3.6 \pm 6.9$ & $-1.3 \pm 5.7$ & $2.5 \pm 5.6^{\star}$ & $<0.001$ \\
\hline$\Delta \mathrm{LV}$ mass index, $\mathrm{g} / \mathrm{m}^{2}$ & $-30.4 \pm 55.6$ & $-14.3 \pm 48.6$ & $-11.2 \pm 45.3$ & 0.193 \\
\hline $\begin{array}{l}\Delta \text { LA volume index, } \\
\mathrm{ml} / \mathrm{m}^{2}\end{array}$ & $-13.4 \pm 28.9$ & $-5.7 \pm 14.6^{\star}$ & $0.2 \pm 18.1^{\star}$ & 0.004 \\
\hline
\end{tabular}

${ }^{*} P<0.05$ compared with the AR regression group.

${ }^{\#} P<0.05$ compared with the AR unchanged group.

$A R$, aortic regurgitation; $L V E D D$, left ventricular end-diastolic diameter; LVESD, left ventricular end-systolic diameter; $L V E F$, left ventricular ejection fraction; $L V$, left ventricle; $L A$, left atrium; Ele', ratio of early diastolic mitral velocity to early diastolic mitral annular velocity; PASP; pulmonary arterial systolic pressure; AV, aortic valve.

\section{Study Limitations}

First, this was a retrospective study and a significant number of patients were excluded because there was no echocardiographic follow-up within a specific time period after KT. Moreover, post-KT echocardiography was not performed according to the pre-specified period. However, we attempted to overcome these shortcomings by including a large number of patients from multiple centers who had undergone
TABLE 6 | Factors associated with progression of aortic regurgitation after KT.

\begin{tabular}{|c|c|c|c|c|c|c|}
\hline & \multicolumn{3}{|c|}{ Univariate analysis } & \multicolumn{3}{|c|}{ Multivariate analysis } \\
\hline & $B$ & $t$ & $p$-value & $B$ & $t$ & $p$-value \\
\hline Age & 0.002 & 1.80 & 0.073 & -0.002 & -0.63 & 0.533 \\
\hline Female sex & 0.040 & 1.47 & 0.142 & & & \\
\hline Hypertension & 0.018 & 0.41 & 0.686 & & & \\
\hline Diabetes mellitus & 0.028 & 0.97 & 0.331 & & & \\
\hline Dyslipidemia & -0.045 & -1.13 & 0.190 & & & \\
\hline Atrial fibrillation & -0.043 & -0.70 & 0.485 & & & \\
\hline CAD & -0.012 & -0.30 & 0.766 & & & \\
\hline Heart failure & -0.010 & -0.21 & 0.832 & & & \\
\hline HD duration & -0.042 & -0.78 & 0.435 & & & \\
\hline New-onset HTN & -0.035 & -0.53 & 0.594 & & & \\
\hline Second KT & 0.042 & 0.82 & 0.415 & & & \\
\hline Persistent HTN & 0.063 & 2.07 & 0.039 & 0.200 & 2.49 & 0.014 \\
\hline New-onset DM & -0.042 & -1.08 & 0.282 & & & \\
\hline Renal dysfunction & 0.043 & 1.25 & 0.214 & & & \\
\hline Graft failure & 0.061 & 1.45 & 0.149 & & & \\
\hline AV calcification & 0.330 & 2.66 & 0.009 & 0.274 & 0.12 & 0.029 \\
\hline$\Delta$ LVEDD & -0.002 & -1.11 & 0.269 & & & \\
\hline$\Delta$ LVESD & 0.006 & 2.73 & 0.007 & 0.010 & 2.01 & 0.046 \\
\hline$\Delta$ LVEF & -0.003 & -2.46 & 0.014 & & & \\
\hline$\Delta \mathrm{LV}$ mass index & 0.027 & 0.52 & 0.603 & & & \\
\hline$\Delta$ LA volume index & 0.002 & 2.21 & 0.028 & & & \\
\hline
\end{tabular}

$C A D$, coronary artery disease; HD, hemodialysis; KT, kidney transplantation; HTN, hypertension; DM, diabetes mellitus; $A V$, aortic valve; $L V E D D$, left ventricular end-diastolic diameter; LVESD, left ventricular end-systolic diameter; LVEF, left ventricular ejection fraction; $L V$, left ventricle; $L A$, left atrium.

follow-up echocardiography between 6 and 36 months after KT.

Second, in this KT cohort, there were many cases of no or mild valve regurgitation before KT. If there was significant or severe valve regurgitation, it is possible that $\mathrm{KT}$ was postponed or not performed, and volume control by dialysis was attempted. Accordingly, there were many patients whose MR and AR did not change after KT. There was a limitation of the population in evaluating the dynamic change of AR and MR. Therefore, it is possible that we selected only relatively healthy ESRD patients. Nevertheless, we believe that we identified patient characteristics that reflect real clinical practice and the natural course of $\mathrm{MR}$ and AR.

Third, although we aimed to find factors that could influence progression of $\mathrm{MR}$ and $\mathrm{AR}$, it is possible that some factors that were not investigated or that undetected confounding factors had an influence on the results.

\section{CONCLUSIONS}

Among patients undergoing $\mathrm{KT}, \mathrm{MR}$, and $\mathrm{AR}$ can progress in those patients with certain characteristics. Risk factors for progression of MR after KT include a second $\mathrm{KT}$, MAC and a smaller decrease in LVESD after KT. Risk factors for progression of AR include valve calcification, persistent hypertension and a smaller decrease in LVESD after KT. Further echocardiographic 
surveillance and risk factor management after $\mathrm{KT}$ are warranted in these patients.

\section{DATA AVAILABILITY STATEMENT}

The original contributions presented in the study are included in the article/Supplementary Material, further inquiries can be directed to the corresponding author.

\section{ETHICS STATEMENT}

The studies involving human participants were reviewed and approved by Institutional Review Board of the Yonsei University Health System (2021-0994-001). Written informed consent for participation was not required for this study in accordance with the national legislation and the institutional requirements.

\section{REFERENCES}

1. Marwick TH, Amann K, Bangalore S, Cavalcante JL, Charytan DM, Craig JC, et al. Chronic kidney disease and valvular heart disease: conclusions from a kidney disease: improving global outcomes (KDIGO) controversies conference. Kidney Int. (2019) 96:836-49. doi: 10.1016/j.kint.2019.06.025

2. Forman MB, Virmani R, Robertson RM, Stone WJ. Mitral anular calcification in chronic renal failure. Chest. (1984) 85:367-71. doi: 10.1378/chest.85.3.367

3. Straumann E, Meyer B, Misteli M, Blumberg A, Jenzer HR. Aortic and mitral valve disease in patients with end stage renal failure on long-term haemodialysis. Br Heart J. (1992) 67:236-9. doi: 10.1136/hrt.67.3.236

4. Samad Z, Sivak JA, Phelan M, Schulte PJ, Patel U, Velazquez EJ. Prevalence and outcomes of left-sided valvular heart disease associated with chronic kidney disease. J Am Heart Assoc. (2017) 6:44. doi: 10.1161/JAHA.117.006044

5. Cirit M, Ozkahya M, Cinar CS, Ok E, Aydin S, Akçiçek F, et al. Disappearance of mitral and tricuspid regurgitation in haemodialysis patients after ultrafiltration. Nephrol Dial Transplant. (1998) 13:38992. doi: 10.1093/oxfordjournals.ndt.a027835

6. Abbott KC, Hshieh P, Cruess D, Agodoa LY, Welch PG, Taylor AJ, et al. Hospitalized valvular heart disease in patients on renal transplant waiting list: incidence, clinical correlates and outcomes. Clin Nephrol. (2003) 59:7987. doi: 10.5414/CNP59079

7. Rangaswami J, Mathew RO, Parasuraman R, Tantisattamo E, Lubetzky M, Rao S, et al. Cardiovascular disease in the kidney transplant recipient: epidemiology, diagnosis and management strategies. Nephrol Dial Transplant. (2019) 34:760-73. doi: 10.1093/ndt/gfz053

8. Ponikowski P, Voors AA, Anker SD, Bueno H, Cleland JGF, Coats AJS, et al. (2016). ESC Guidelines for the diagnosis and treatment of acute and chronic heart failure: the task force for the diagnosis and treatment of acute and chronic heart failure of the European Society of Cardiology (ESC)Developed with the special contribution of the Heart Failure Association (HFA) of the ESC. Eur Heart J. (2016) 37:2129-200. doi: 10.1093/eurheartj/ehw128

9. Thomas ME, Blaine C, Dawnay A, Devonald MA, Ftouh S, Laing C, et al. The definition of acute kidney injury and its use in practice. Kidney Int. (2015) 87:62-73. doi: 10.1038/ki.2014.328

10. Cheitlin MD, Armstrong WF, Aurigemma GP, Beller GA, Bierman FZ, Davis JL, et al. ACC/AHA/ASE (2003). Guideline update for the clinical application of echocardiography: summary article. a report of the American College of Cardiology/American Heart Association Task Force on Practice Guidelines (ACC/AHA/ASE Committee to Update the (1997). Guidelines for the Clinical Application of Echocardiography). J Am Soc Echocardiogr. (2003). 16:1091110. doi: 10.1016/S0894-7317(03)00685-0

11. Mitchell C, Rahko PS, Blauwet LA, Canaday B, Finstuen JA, Foster $\mathrm{MC}$, et al. Guidelines for performing a comprehensive transthoracic echocardiographic examination in adults: recommendations from the american society of echocardiography. J Am Soc Echocardiogr. (2019) 32:1-64. doi: 10.1016/j.echo.2018.06.004

\section{AUTHOR CONTRIBUTIONS}

MK reviewed the literature and wrote the first draft of the manuscript. CS supervised this paper, coordinated the multidisciplinary discussion, and critically reviewed the manuscript. DK provided the data of the Samsung medical center and reviewed the manuscript. JL, D-YK, JS, IC, $\mathrm{KH}, \mathrm{G}-\mathrm{RH}$, and $\mathrm{J}-\mathrm{WH}$ reviewed the manuscript, provided comments, and suggested modifications to the manuscript. All authors contributed to the article and approved the submitted version.

\section{SUPPLEMENTARY MATERIAL}

The Supplementary Material for this article can be found online at: https://www.frontiersin.org/articles/10.3389/fcvm. 2022.809707/full\#supplementary-material

12. Zoghbi WA, Adams D, Bonow RO, Enriquez-Sarano M, Foster E, Grayburn PA, et al. Recommendations for noninvasive evaluation of native valvular regurgitation: a report from the American Society of Echocardiography Developed in collaboration with the society for cardiovascular magnetic resonance. J Am Soc Echocardiogr. (2017) 30:303-71. doi: 10.1016/j.echo.2017.01.007

13. Kim D, Shim CY, Hong GR, Jeong H, Ha JW. Morphological and functional characteristics of mitral annular calcification and their relationship to stroke. PLoS ONE. (2020) 15:e0227753. doi: 10.1371/journal.pone.0227753

14. Kohsaka S, Jin Z, Rundek T, Boden-Albala B, Homma S, Sacco RL, et al. Impact of mitral annular calcification on cardiovascular events in a multiethnic community: the Northern Manhattan Study. JACC Cardiovasc Imag. (2008) 1:617-23. doi: 10.1016/j.jcmg.2008.07.006

15. Lu ML, Gupta S, Romero-Corral A, Matejková M, De Venecia T, Obasare E, et al. Cardiac calcifications on echocardiography are associated with mortality and stroke. J Am Soc Echocardiogr. (2016) 29:11718. doi: 10.1016/j.echo.2016.08.020

16. Lu KC, Wu CC, Yen JF, Liu WC. Vascular calcification and renal bone disorders. Sci World J. (2014) 2014:637065. doi: 10.1155/2014/637065

17. Neale J, Smith AC. Cardiovascular risk factors following renal transplant. World J Transpl. (2015) 5:183-95. doi: 10.5500/wjt.v5.i4.183

18. Jesri A, Braitman LE, Pressman GS. Severe mitral annular calcification predicts chronic kidney disease. Int J Cardiol. (2008) 128:193-6. doi: 10.1016/j.ijcard.2007.05.015

19. Rahimi K, Mohseni H, Kiran A, Tran J, Nazarzadeh M, Rahimian F. et al. Elevated blood pressure and risk of aortic valve disease: a cohort analysis of 54 million UK adults. Eur Heart J. (2018) 39:3596603. doi: 10.1093/eurheartj/ehy486

Conflict of Interest: The authors declare that the research was conducted in the absence of any commercial or financial relationships that could be construed as a potential conflict of interest.

Publisher's Note: All claims expressed in this article are solely those of the authors and do not necessarily represent those of their affiliated organizations, or those of the publisher, the editors and the reviewers. Any product that may be evaluated in this article, or claim that may be made by its manufacturer, is not guaranteed or endorsed by the publisher.

Copyright (C) $2022 \mathrm{Kim}$, Kim, Lee, Kim, Seo, Cho, Huh, Hong, Ha and Shim. This is an open-access article distributed under the terms of the Creative Commons Attribution License (CC BY). The use, distribution or reproduction in other forums is permitted, provided the original author(s) and the copyright owner(s) are credited and that the original publication in this journal is cited, in accordance with accepted academic practice. No use, distribution or reproduction is permitted which does not comply with these terms. 